\title{
A Point Projection Microscope for Electron Interferometry in the Reflection Mode
}

\author{
B.G.Frost, A.Thesen and D.C.Joy \\ University of Tennessee, M407 Walters Life Sci, Knoxville, TN 37996-0840
}

We designed a new microscope for the metrology of sub-micron design rule devices. This tool combines the principles of a point projection microscope $(\mathrm{ppm})$ with the ones of a mirror electron microscope (mem). The original ppm works only in the transmission mode and consists of an electron point emitter [1], a sample and an electron detector. The negatively biased nano-tip point source delivers an ultra low energy electron (50-250eV) current at several nanoamps. The electrons are extracted by the grounded sample which is placed several microns from the tip. In the image plane the highly coherent electrons interfere and form an in-line hologram. The inventors [2] of the ppm describe their new tool as follows: "As this type of microscope involves no electron- optical lens elements, the images obtained are free from the ordinary aberrations. The limit of resolution depends solely on the distribution of initial velocities of the field electrons and on Fresnel diffraction by the object, making it possible to proceed beyond the resolution of the light microscope by some orders of magnitude." Figure 1 shows a ray diagram of a ppm, a holey carbon foil acquired with this set-up is shown in figure 2.

In order to apply the ppm to bulk materials we set-up a special reflection mode of operation.

When we apply the principle of the ppm to the reflection mode we have to add the basic ideas of a mem. In order to increase the electron reflectivity at the sample surface we negatively bias the sample which then does not act as anode and no electrons are extracted from the tip. Thus the tip has to be replaced by an electron gun. Using this set-up we acquired images of a fine copper mesh and compare the results to our simulations. It turns out that the resolved surface structures strongly depend on the orientation between sample and electron gun if the beam is not normally incident on the surface. This can be seen by comparing figure 3 with figure 4 where the specimen was rotated by 45 degrees. We discuss a special set-up which is similar to a field ion microscope and suitable for atomic resolution.

\section{References}

[1] H.W. Fink, IBM J. Res. Develop. 30 (1986) 460.

[2] G.A. Morton and E.G.Ramberg, Phys.Rev. 56 (1939) 705.

[3] This research was supported by SRC under contract number LJ413.003 and was carried out as part of the user program sponsored by the U.S. Department of Energy under contract DE-AC05-00OR22725 with the Oak Ridge National Laboratory, managed by UT-Battelle, LLC. 


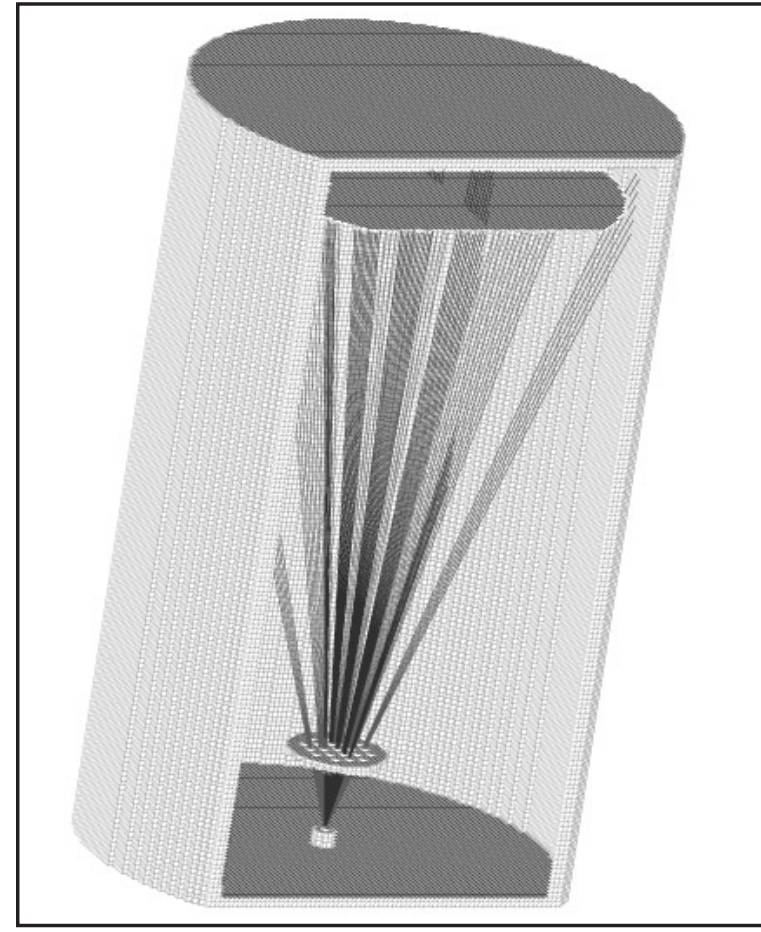

FIG.1. Ray diagram of low voltage point projection microscope.

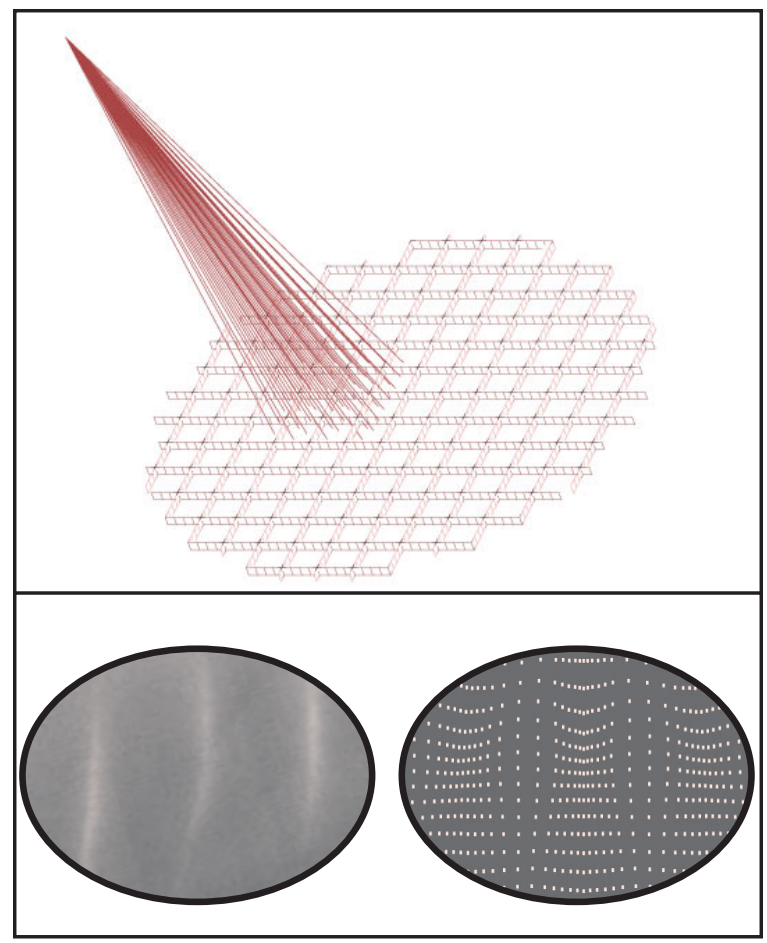

FIG.3. Fine copper mesh acquired in reflection mode of ppm.

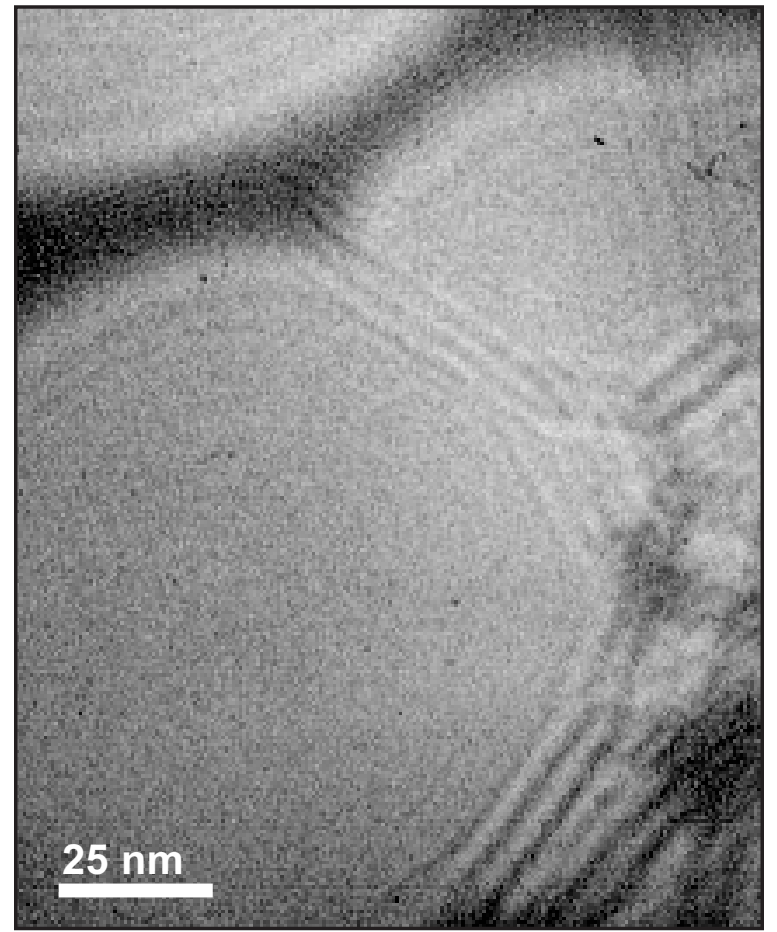

FIG.2. In-line hologram of carbon foil acquired in transmission mode of ppm.

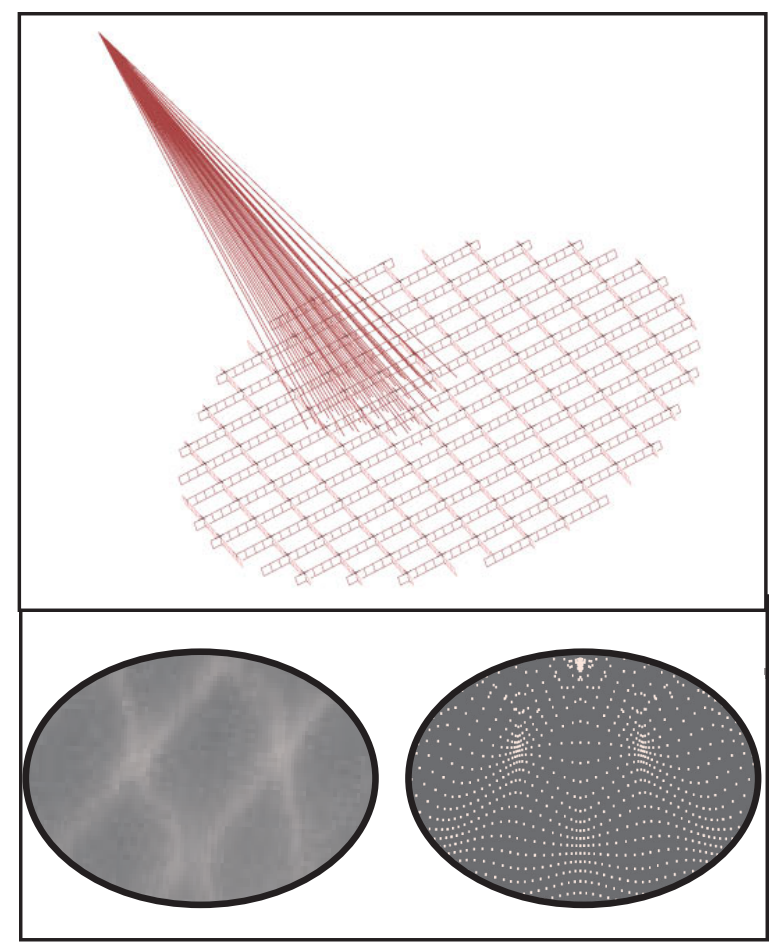

FIG.4. Same as figure 3. Sample rotated by 45 degrees. 